\title{
ELECTRICAL EXCITATION OF THE NERVE FIBER PART II. EXCITATION BY EXPONENTIALLY INCREASING CURRENTS
}

\author{
ICHIJI TASAKI AND MITSUNADA SAKAGUCHI' \\ Physiological Institute, Keio University, Yotsuya, Tokyo
}

In a preceding paper, one of us reported the results of experiments dealing with excitation of isolated single nerve fibers by linearly increasing currents. In the present paper, we are concerned with the problem of excitation of the nerve fiber by exponentially increasing currents. This problem has already been treated by many previous workers with the nerve trunk and the muscle (Gildemeister (3), Cardot and Laugier (1), Lapicque (5), Schriever (6), Suzuki (8), Solandt (7), and others). We have thoroughly reinvestigated this problem with isolated single nerve fibers.

\section{METHOD}

The material and the apparatus used were the same as in the previous experiments (Tasaki (9)). The electric response of a single motor nerve fiber was taken in most cases as index of nerve excitation. In some cases, muscular contraction of the all-or-none character was taken as index. Circuits used for applying exponentially increasing voltage were those shown in Fig. 1 in the previous paper and in Fig. 1 in the present paper. Circuit A in Fig. 1 was
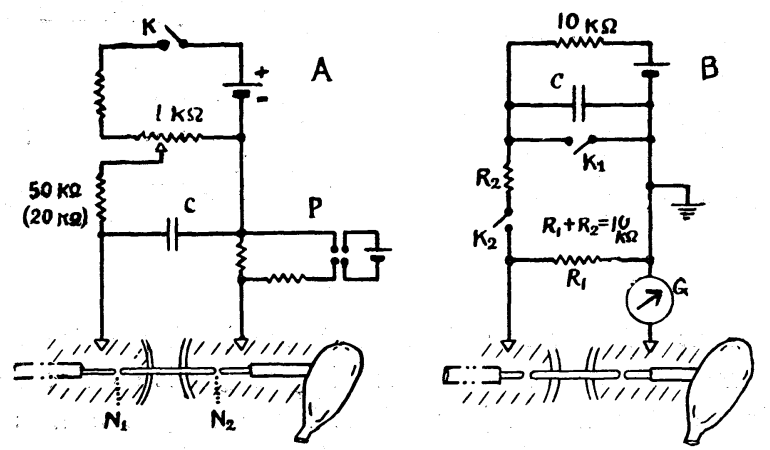

Fig. 1. Circuit used for applying exponentially rising voltage to a toad's motor nerve fiber. Circuit $\mathrm{P}$ was used for applying electrotonic current to the fiber. $\mathrm{K}$ represents a mercury-copper contact; $\mathrm{K}_{\mathrm{r}}$ and $\mathrm{K}_{2}$, break contacts of a Lucas pendulum. The source of current was an accumulator of about 2 volts.

1 田崎一二, 坂口光洋

Received for Publication September 26, 1944 by the redactor of the Japanese Journal of Medical Sciences (Biophysics). 
the simplest one: the contact $\mathrm{K}$ was closed for about 1 second and the threshold was determined taking make-excitation of the nerve fiber as index. Circuit $B$ in Fig. 1 was used for determining the utilization-time (Nutzzeit) in excitation with exponentially rising voltage: opening the contact $K_{1}$ of a Lucas pendulum started the voltage-rise and opening $K_{2}$ decreased the voltage suddenly to zero. The capacity of the condenser was varied from 0 up to $20 \mu \mathrm{F}$.

The time-constant of voltage-increase was given, in the circuit A in Fig. 1, by the product of $R(=50$ or $20 \mathrm{~K} \Omega$.) into $C$. In the circuit $\mathrm{B}$, it was given by the product of $5 \mathrm{~K} \Omega$. and the value of $C$. As the preparation was laid on a bridge-insulator, the resistance of the preparation was of the order of $50 \mathrm{meg}$ ohms. The final value of the voltage was read directly in the potential-divider of $1 \mathrm{~K} \Omega$. (in A) or $10 \mathrm{~K} \Omega$ (in $\mathrm{B}$ ).

Circuit $\mathrm{P}$ in Fig. 1 was used for applying electrotonic currents to the nerve fiber ; a descending current made the node of Ranvier $\mathrm{N}_{2}$ on the distal side of the bridge-insulator catelectrotonic, and an ascending current made this node anelectrotonic.

When the latent period of action current was to be measured, the base-line of the figure on the screen of the cathode-ray tube was distorted with an oscillator of 1000 cycles per second. Fig. 2 shows several examples of the records of action currents of a nerve fiber elicited by application of exponentially rising voltages of varying time-constants. The final voltages were near threshold in all cases.
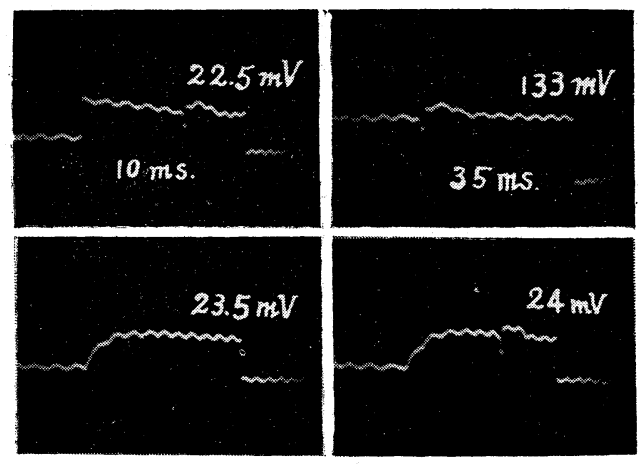

Fig. 2. Electric responses of a motor nerve fiber of the toad, elicited by application of a rectangular current pulse of the duration of $10 \mathrm{msec}$. (top left) and of exponentially rising currents of the time-constants $(R C)$ of $1 \mathrm{msec}$. (bottom) and $100 \mathrm{msec}$. (top right). The final voltages are given in the figure. In the record at the top, right, the stimulating voltage was interrupted after 35 msec. from the beginning of the stimulus. $17^{\circ} \mathrm{C}$.

RESULTS

(1) The relation between the time-constant of voltage-rise and the threshold. The relation between the time-constant of voltage-rise $(R \times C)$ and the final value of the voltage $\left(V_{i}\right)$ which just elicited the response was investigated in the first place. An example of the results is given in Table $I$ and is illustrated in Fig. $4 \mathrm{~B}$, (1). In the last column of the table, values calculated by assuming a linear relationship between $V_{i}$ and $R C$ are given. In this case muscular contraction was taken as index.

In all the results, the relation between $V_{i}$ and $R C$ showed a striking linearity, which could be expressed by the equation

$$
V_{i}=(1+m R C) b \text {. }
$$


Table I

\begin{tabular}{c|rc|c}
\hline$R C(\mathrm{msec})$ & \multicolumn{2}{|c|}{$V_{i}(\mathrm{mV})}$. & $(1+R C / 88) 33.5$ \\
\hline 0 & $32-34$ & $32-34$ & 33.5 \\
17.7 & $39-41$ & - & 40.3 \\
36.0 & $45-47$ & $47-50$ & 47.2 \\
0 & $33-34$ & - & - \\
76 & $62-65$ & $60-62$ & 62.6 \\
158 & $92-95$ & $98-100$ & 93.6 \\
236 & $120-125$ & $120-125$ & 124.0 \\
0 & - & $33-35$ & - \\
\hline
\end{tabular}

Table II shows the values of $b$ and $1 / m$ in the experimental results obtained by one of us, Tasaki, (during January of 1943) by using the experimental arrangements of Fig. $1 \mathrm{~A}$. The rheobasic voltage $b$ was in most cases between 20 and $40 \mathrm{mV}$., and the value of $1 / m$, which corresponds Hill's time-constant of accomodation ( $\lambda$ ), was between about 40 and $150 \mathrm{msec}$. The content of $\mathrm{CaCl}_{2}$ in the Ringer solution was, as in the previous experiments, 0.12 gram in 1 litre and that of $\mathrm{KCl}$ was 0.14 gram.

Table II

\begin{tabular}{|c|c|c|c|c|c|}
\hline$b(\mathrm{mV} \cdot)$ & $.1 / m$ (msec.) & temperature & $b(\mathrm{mV}$.$) .$ & $1 / m$ (msec.) & tempereture \\
\hline 25 & 51 & $10^{\circ} \mathrm{C}$ & 27 & 38 & $9^{\circ} \mathrm{C}$ \\
\hline 31 & 65 & 10 & 22 & 50 & 9 \\
\hline 33 & 88 & 10 & 22 & 75 & 9 \\
\hline 25 & 153 & 9 & 19 & 36 & 10 \\
\hline 25 & 125 & 6 & 21 & 64 & 7 \\
\hline 39 & 84 & 6 & 35 & 155 & 7 \\
\hline 29 & 41 & 8 & 22 & 52 & 7 \\
\hline 25 & 80 & 8 & 23 & 88 & 6 \\
\hline 31 & 91 & 8 & 18 & 53 & 5 \\
\hline 24 & 120 & 8 & 40 & 71 & 9 \\
\hline 35 & 60 & 8 & 41 & 58 & 9 \\
\hline 32 & 66 & 8 & 29 & 54 & 10 \\
\hline
\end{tabular}

The variation in the value of $1 / m$ among different preparations was more marked than that in the rheobasic voltage $b$. In the experiments done between November of 1941 and April of 1942, we have met with several cases in which $1 / m$ was below 20 msec.

Another example of the experimental results obtained by using the arrangements of Fig. $1 \mathrm{~B}$ is given in Table III and is partially illustrated in Fig. 3. The first and the second column in the table show, as in Table $I$, the values of $R C$ and corresponding threshold strength $V_{i}$. The third column $(l)$ shows the observed values of the latent periods of action currents in threshold excitation, i.e. the time-interval between the beginning of the exponentially rising voltages 
Table III

\begin{tabular}{r|c|ccc|c|c}
\hline \hline$R C$ (msec.) & $V_{i}$ (mV.) & \multicolumn{2}{|c|}{$l$ (msec.) } & $\tau$ (msec.) & $\begin{array}{c}\frac{d V(t)}{d t} \\
\text { at } t=\tau\end{array}$ \\
\hline 0 & $30-31$ & 3.5, & 4 &. & - & - \\
10 & $44-45$ & 16, & 14 &. & 11.6 & 13.9 \\
20 & $54-55$ & 20, & 26, & 19 & 16.4 & 12.0 \\
30 & $66-68$ & 25, & 21 & & 18.2 & 12.2 \\
40 & $80-82$ & 26, & 27, & 24 & 18.9 & 12.5 \\
50 & $94-96$ & 24, & 23, & 27 & 19.3 & 12.9 \\
70 & $120-122$ & 32, & 22 & & 20.2 & 13.0 \\
100 & $160-165$ & 29, & 27,29, & 27 & 20.6 & 13.0 \\
\hline
\end{tabular}

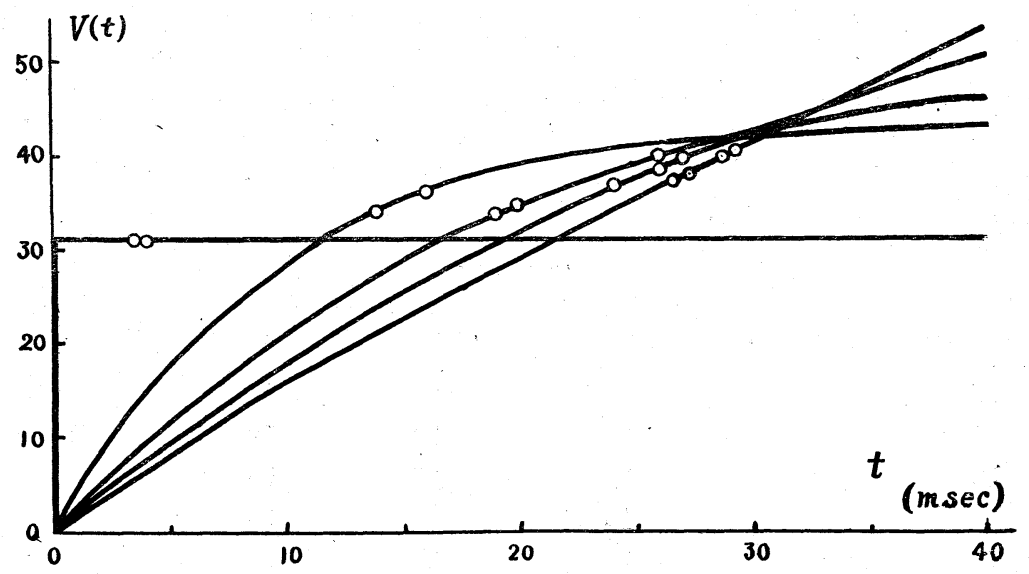

Fig. 3. Diagram showing the time-course of exponentially rising voltages by which a nerve fiber was just excited. The data of Table III. The circles indicate the moments at which action currents of the fiber was observed. $16^{\circ} \mathrm{C}$.

of the threshold strengths and the electric responses at the site of stimulation. In the figure, the temporal configuration of the applied voltages

$$
V(t)=V_{i}\left(1-e^{-t / R C}\right)
$$

are shown with continuous curves. The final values of the voltage $\left(V_{i}\right)$ in these cases correspond to the threshold values at that value of $R C$. The circles in the figure indicate the moments, at which electric responses of the nerve fiber were observed, when the fiber was excited by these exponentially rising voltages of the threshold strengths.

It is clearly shown in the figure that the electric response of the nerve fiber appears slightly $(2 \sim 12 \mathrm{msec}$.) after the applied voltage has reached the rheobasic voltage. In the third column of the table, the time is given at which the exponentially rising voltage of the threshold strength $V_{i}$ (the mean of the two $\mathrm{va}^{\top}$ ues in the second column) reaches the rheobase, calculated by introducing the corresponding values of $R C$ and $V_{i}$ into 


$$
\tau=R C \times 2.30 \times \log \left\{V_{i} /\left(V_{i}-30.5\right)\right\} .
$$

The fourth column gives the voltage-gradient at $t=\tau$, calculated by the relation

$$
\frac{d V(t)}{d t}=\frac{V_{i}}{R C \times 10^{t /(2,30 \cdot R C)}}
$$

It is to be noticed in Table III and Fig. 2, that the voltage-gradient at the moment $t=\tau$ is practically constant, regardless of the value of $R C$. This fact is however nothing worthy of surprise. It is a direct consequence of the experimental fact, that the relation between the time-constant $R C$ and the corresponding $V_{i}$ is linear.

By differentiating the equation (2), representing the course of the exponentially rising voltage of the threshold strength, we obtain

$$
\begin{aligned}
\frac{d V(t)}{d t} & =V_{i} \frac{1}{R C} e^{-t / R C} \\
& =\frac{1}{R C}\left(V_{i}-V(t)\right) .
\end{aligned}
$$

Introducing the experimentally verified relation of Eq. (1) into Eq. (3'), we obtain further

$$
\frac{d V(t)}{d t}=m b+\frac{b-V(t)}{R C} .
$$

This last relation shows that, when a nerve fiber is excited with exponentially rising voltages of the threshold strengths, the gradient of the voltage $d V(t) / d t$ takes a constant value $m b$ at the moment when $V(t)$ reaches the rheobasic voltage $b$, regardless of the value of $R C$.

When the time-constant of voltage-rise $R C$ is sufficiently great, the voltage rises almost linearly during the early period of voltage-rise (see Fig. $2, R C=100$ ). Eq. (4) indicates that the rate of voltage-rise in threshold excitation approaches a definite value $m b$ of that fiber as $R C$ increases. It is therefore evident, that the value $m b$ is nothing but the minimal voltage gradient in excitation of the fiber with linearly rising currents. The experimental results described above can consequently be stated as indicating that exponentially rising voltages of the threshold strengths cross the rheobasic voltage at a constant gradient which is equal to the minimal voltage gradient of the fiber.

An exponentially rising voltage of just subthreshold strength can be made effective to elicit electric response either by increasing the final value of the voltage $V_{i}$ or by decreasing the time-constant of current rise $R C$; these procedures in turn increases, according to Eq. (3), the gradient of the voltage in the early period of voltage-rising. It is therefore clear that an exponentially rising voltage excites the fiber if it crosses the rheobasic voltage at a rate greater than the minimal gradient of that fiber.

In a preceding paper, one of us has concluded, that a linearly increasing voltage excites the nerve fiber if it rises above the rheobasic voltage at a rate greater than the minimal gradient of the fiber. This conclusion was now extended to include the exponentially rising voltages. The electric response of the fiber was found to be evoked, as in the previous experiments, some period 
after the stimulating voltage has risen beyond the rheobase. The length of this period was generally between 2 and $10 \mathrm{msec}$; it showed remarkable variation when the final voltage $V_{i}$ approached the threshold value at that value of $R C$.

(2) The utilization-time. In this series of experiments the circuit B in Fig. 1 was used. The capacity of the condenser $C$ in the figure, and consequently the value of $R C$, was fixed at a constant value during one series of experiment. The final voltage $V_{i}$, which was regulated by the potential divider, was increased above the liminal value at that $R C$, and the period of the voltage-rise, which was controlled by means of break-contacts $K_{1}$ and $K_{2}$ of the Lucas pendulum, was decreased step by step until finally the response of the fiber just failed to appear.

An example of the results of such an observation is given in Table IV. The capacity of the condenser (circuit B of Fig. 1) was $20 \mu \mathrm{F}$. in this case; as the resistance of the circuit was $5 \mathrm{~K} \Omega$., the time-constant of voltage-rise was in this case $100 \mathrm{msec}$. The muscular contraction was taken as index. 'The symbol " $50-53$ " in the table means that, at $R C=100 \mathrm{msec}$. and $V_{i}=110 \mathrm{mV}$., the exponentially rising voltage was effective to excite the fiber when the stimulating

Table IV

\begin{tabular}{l|cc|c}
\hline \hline$V_{i}(\mathrm{mV})$. & $T$ (msec.) & $V(T)(\mathrm{mV})$. \\
\hline 105 & no response & - \\
110 & $50-53$ & $51-53$ & $43.5-45$ \\
130 & $34-36$ & $37-39$ & $-37.5-41.5$ \\
150 & $30-32$ & $30-31$ & $39-40$ \\
170 & $24-26$ & $25-27$ & $36-40$ \\
200 & $20-22$ & $20-22$ & $38-40$ \\
250 & $16-17$ & - & $37-39$ \\
\hline \multicolumn{2}{r}{} \\
\end{tabular}

voltage was allowed to act upon the fiber for a period of $53 \mathrm{msec}$. from the beginning of the condenser-charge, and that when this period was reduced to 50 msec. the fiber was found to fail to respond. The values of $V(T)$ in the table show the voltage acting upon the fiber at the end of this utilization-time $T$; these values were obtained by introducing into Eq. (2) the corresponding values of $T$. The rheobasic voltage of the fiber is also given in the table.

The result of these experiments indicated clearly that the utilization-time $T$ is slightly longer than the time $\tau$ at which the stimulating voltage crosses the rheobase. In the observations in which electric responses of the fiber were taken as index, it was found that the utilization-time $T$ was approximately equal to the latent period $l$, the time-interval from the onset or stimulation to the appearance of the response.

(3) Effect of calcium and electrotonus. A change in $\mathrm{CaCl}_{2}$ content in the Ringer solution invariably changed the rheobasic voltage of the nerve fiber: an increase thereof raised and a decrease lowered the rheobase. But, the minimal 
gradient $m$ remained thereby almost uninfluenced. Fig. 4 illustrates two examples of the results we obtained. In $\mathrm{A}$ in this figure, the $\mathrm{CaCl}_{2}$-content was increased by mixing normal Ringer with isotonic $\mathrm{CaCl}_{2}$ solution at a proportion of $1: 4$. In $\mathrm{B}$ the $\mathrm{CaCl}_{2}$-content was decreased by mixing normal Ringer with $\mathrm{CaCl}_{2}$-deficient Ringer. In these cases, threshold determination was commenced few

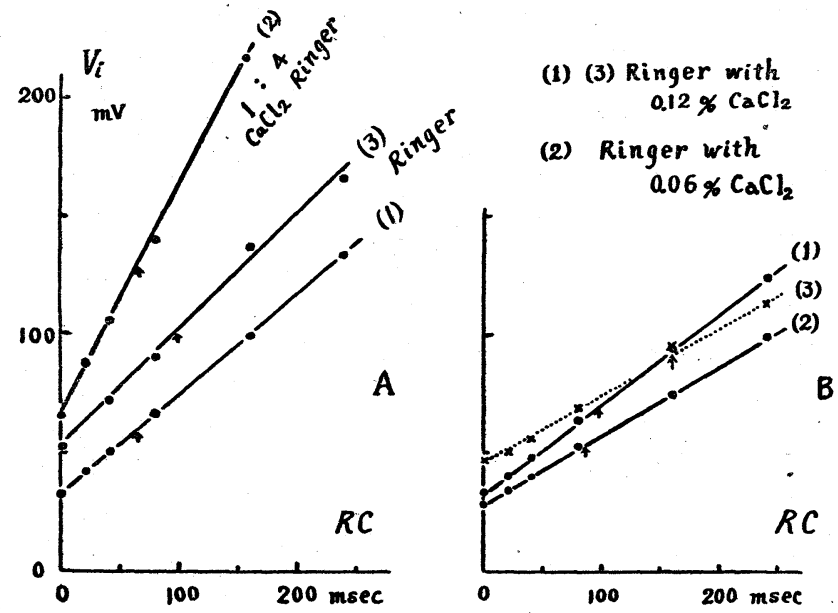

Fig. 4. Effect of $\mathrm{CaCl}_{2}$ upon the rheobase and the minimal gradient of a single nerve fiber. The arrows indicate the reciprocals of the minimal gradient, which correspond to Hill's constant of accommodation. A was obtained at $6^{\circ} \mathrm{C}$, and $\mathrm{B}$ at $10^{\circ} \mathrm{C}$.

minutes after replacing the normal Ringer with these abnormal solutions. The preparations were finally soaked in the normal Ringer again and the relation between $V_{i}$ and $R C$ was determined (curves 3 in the figures); by this procedure however the rheobasic voltage and the minimal gradient of the fiber did not as a rule return to their original values which the fiber showed when it was primarily in the normal Ringer.

When a cathodally polarizing voltage of several ten millivolts was applied to the nerve fiber, the rheobase was found to be suddenly lowered by this procedure, and then it rose gradually during a period of about 10 minutes. In the experiments described in this paper, determination of the $V_{i}-R C$ curve was done during the period from 15 to 40 minutes after the onset of electrotonus. At this stage of catelectrotonus, the rheobasic voltage was approximately equal to its original value. In the case of anelectrotonus, the rheobase showed a high value depending on the strength of the polarizing voltage applied.

Fig. 5 gives an example of the results obtained. Application of a cathodally polarizing voltage (C.E.T.) of $60 \mathrm{mV}$. has increased the minimal gradient from the normal value of $1 / 62 \mathrm{msec}^{-1}$ to $1 / 18$; the rheobase was not appreciably changed in this case. An anodal polarization with a voltage of $60 \mathrm{mV}$. has increased the rheobase from the normal value of $25 \mathrm{mV}$. up to $100 \mathrm{mV}$., and the minimal gradient was thereby decreased to about $1 / 900 \mathrm{msec}^{-1}$ 


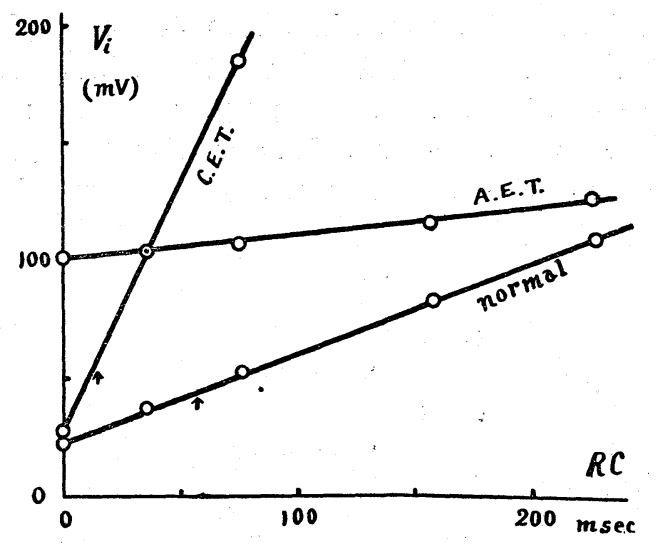

Fig. 5. Effect of electrotonus $(60 \mathrm{mV}$.) upon the rheobase and the minimal gradient of a single nerve fiber. All the data were obtained for one and the same preparation. Measurements were begun after about 20 minutes from the onset of electrotonus. $5^{\circ} \mathrm{C}$.

\section{DISCUSSION}

In the preceding paper (9), we have seen that a linearly increasing voltage excites a nerve fiber if it rises above the rheobasic voltage of the fiber at a rate greater than the minimal gradient of the fiber. The results of the present investigation indicate that we can generalize the conclusion stated above in the following form:

Let a slowly increasing voltage, applied to a nerve fiber, of which the time course is given by $V(t)$, surpass the rheobase $b$ at the moment $t=\tau$; then, the rate of voltage increase at this moment must be greater than $m b$ if the voltage is to elicit a response from the fiber. In threshold excitation, the two conditions

$$
V(t)=b \text {. }
$$

and

$$
\frac{d V(t)}{d t}=m b
$$

are simultaneously satisfied at the moment $t=\tau$.

Introducing the relation of Eq. (2) into these simultaneous equations and eliminating the time, we readily obtain Eq. (1) which represents the linear relationship between the final voltage $V_{i}$ and the time-constant of voltage-rise $R C$ in excitation by exponentially rising voltages.

\section{SUMMARY}

(1) Isolated single nerve fibers of the toad were excited with exponentially rising voltages. It was shown that the relation between the final voltage $V_{i}$ and the time-constant of voltage-rise $R C$ is expressed by a good straight line. This experimental fact indicates that the rate of increase of the stimulating voltage $\frac{d V(t)}{d t}$ shows a constant value at the moment when the voltage $V(t)$. crosses the rheobasic voltage.

(2) The utilization-time and the latent period of action current were also determined. It was found that the action current of the nerve fiber is set up a 
few milliseconds after $V(t)$ has crossed the rheobasic voltage at a rate greater than the minimal voltage gradient of the fiber. The utilization-time ended near the moment at which the action current of the fiber was set up.

(3) The effect of $\mathrm{CaCl}_{2}$-excess and $\mathrm{CaCl}_{2}$-deficiency and of electrotonus upon the $V_{i}-R C$ relation was also investigated. Change in the content of $\mathrm{CaCl}_{2}$ in Ringer changed the rheobase but not at once influenced the minimal gradient of the fiber. Catelectrotonus decreased and anelectrotonus increased the minimal gradient.

\section{LITERATURE}

1. Cardot, H. ET LaUgier. J. Physiol. Pathol. Gen. 15: 1134, 1913.

2. Delville, P. Arch. intern. Physiol. 40: 83, 1934.

3. GILDEMEISTER, M. Z. Biol. 62: 358, 1913.

4. HILl, A. V. Proc. Roy. Soc. B. $119:$ 305, 1936.

5. LAPICQUE, L.' L'excitabilité en fonction du temps. Les Presse Universitaires de France 1925.

6. SCHRIEVER, H. Z. Biol. 91 : 173, 1930.

7. SolandT, D. Y. Proc. Roy. Soc. B. 119 : 355, 1936.

8. SUZUKI, M. Pfiügers. Arch. 230, 1932.

9. TASAKI, I. This journal, $1: 1,1950$. 